\title{
High resolution slice-selective Fourier Velocity Encoding using spiral SENSE with velocity unwrap
}

\author{
Jennifer A Steeden ${ }^{1 *}$, David Atkinson², Alexander Jones ${ }^{1}$, Vivek Muthurangu ${ }^{1}$ \\ From 15th Annual SCMR Scientific Sessions \\ Orlando, FL, USA. 2-5 February 2012
}

\section{Background}

In patients with congenital heart disease (CHD), it is desirable to accurately measure peak velocity (Vmax). Unfortunately, phase-contrast MR (PCMR) tends to underestimate peak velocities. Fourier Velocity Encoding (FVE) can measure peak velocities in MRI, but is not commonly used due to long acquisition times.

Therefore, we have developed a FVE sequence that combines spiral trajectories with parallel imaging (SENSE), partial-Fourier acquisition and a novel velocity-unwrap technique. The aim of this study is to validate this sequence.

\section{Methods}

FVE sequence: FVE was performed using a spiral trajectory (table 1). Spiral interleaves were undersampled $(\mathrm{R}=4)$ and reconstructed using an iterative SENSE algorithm. Partial Fourier (67\%) was performed in kv with a homodyne reconstruction was used. The velocityunwrap method purposefully aliases data in $\mathrm{v}$ (by acquiring half the number of kv-positions), and unwrapped using prior information about the flow direction. Peak velocity was determined using previously described techniques 1-3.

In-vitro: A pulsatile flow pump was connected to a tube phantom (13mm diameter) with a stenosis $(6 \mathrm{~mm}$ diameter). Peak velocity measurements using the following techniques were compared at 15 different flow rates; 1) US doppler, 2) low-resolution PCMR (lr-PCMR), 3) high-resolution PCMR (hr-PCMR), 4) FVE.

In-vivo: 12 CHD patients (7M:5F; $34.3 \pm 18.8$ years) with stenoses were assessed. Peak velocity measurements were compared between; 1) lr-PCMR, 2) hrPCMR, and 3) FVE.

\section{Results}

In-vitro: There were no statistically significant differences between Vmax measured using US and FVE (table 2). However both PCMR sequences showed a statistically significant underestimation of peak flow compared to US (table 2). This is particularly true of lr-PCMR, which underestimated Vmax by $>0.5 \mathrm{~m} / \mathrm{s}$. In-vivo: There was a significant underestimation of Vmax measured using both PCMR sequences when compared to FVE (lr-PCMR; $229 \pm 42 \mathrm{~cm} / \mathrm{s}$, hr-PCMR; $238 \pm 46 \mathrm{~cm} / \mathrm{s}$, FVE; $256 \pm 67 \mathrm{~cm} / \mathrm{s}$ ).

\section{Conclusions}

Fourier velocity encoding allows accurate assessment of peak velocities as it measures a velocity spectrum per pixel, rather than the average velocity. However this extra encoding takes time, which has reduced its clinical effectiveness. We have shown that it possible to achieve high resolution FVE within a short breath-hold by combining spiral trajectories, parallel imaging, partial Fourier and velocity-unwrap. This sequence was shown to be significantly more accurate than PCMR in-vitro, and also to provide higher peak velocities than PCMR invivo. Thus, the sequence should be able to replace Doppler echocardiography making CMR a true one-stopshop in assessing congenital heart disease.

\section{Funding}

JAS: EPSRC PhD+.

VM: BHF.

\section{Author details \\ ${ }^{1}$ Institude of Cardiovascular Science, UCL, London, UK. ${ }^{2}$ Centre for Medical Imaging, UCL, London, UK.}

Published: 1 February 2012

Institude of Cardiovascular Science, UCL, London, UK

Full list of author information is available at the end of the article

(c) 2012 Steeden et al; licensee BioMed Central Ltd. This is an open access article distributed under the terms of the Creative Commons Attribution License (http://creativecommons.org/licenses/by/2.0), which permits unrestricted use, distribution, and reproduction in any medium, provided the original work is properly cited. 
Table 1 Imaging Parameters

\begin{tabular}{cccc}
\hline & Ir-PCMR & hr-PCMR & FVE \\
\hline TE/TR (ms) & $\sim 2.2 / 5.0$ & $\sim 2.2 / 5.0$ & $\sim 3.5 / 10.3$ \\
Readouts & Cartesian & Cartesian & $\begin{array}{c}\text { Spiral: } 16 \\
\text { interleaves }\end{array}$ \\
Acceleration factor (in kx-ky) & 2 & 2 & 4 (SENSE) \\
& (GRAPPA) & (GRAPPA) & \\
Matrix Size & 128 & 256 & 192 \\
Image FOV (mm) & 320 & 320 & 450 \\
Total Scan Duration & 15 & 108 & 15 \\
(heartbeats) & & & \\
Spatial Resolution (mm) & $\sim 2.5$ & $\sim 1.3$ & $\sim 2.3$ \\
Temporal Resolution (ms) & $\sim 40$ & $\sim 30$ & $\sim 41$ \\
Velocity Resolution (cm/s) & - & - & $15-38$ \\
\hline
\end{tabular}

\section{Table 2 In-vitro results}

\begin{tabular}{ccccc}
\hline & Echo & Ih-PCMR & hr-PCMR & FVE \\
\hline Peak velocity (cm/s) & 441 & $375 \pm 133 \wedge$ & 398 & $443 \pm 144$ \\
& \pm 144 & & $\pm 136 \wedge$ & \\
Bias* $(\mathrm{cm} / \mathrm{s})^{\text {Limits of Agreement* }}$ & - & -66 & -42 & +3 \\
(cm/s) & & -26 to & -10 to -75 & +17 to \\
$\begin{array}{c}\text { Correlation coefficient* } \\
(\mathrm{r})\end{array}$ & - & -105 & & -12 \\
& & & & \\
\hline
\end{tabular}

* Calculated with echo $\wedge$ Value is significantly different (ANOVA) from echo (P $<0.05)$

\section{References}

1. Hansen MS: Magma. 2004, 17(2):86.

2. Baltes C: Radiology. 2007, 246(1):249.

3. Galea D: Medical Physics. 2002, 29(8):1719.

doi:10.1186/1532-429X-14-S1-O40

Cite this article as: Steeden et al:: High resolution slice-selective Fourier

Velocity Encoding using spiral SENSE with velocity unwrap. Journal of

Cardiovascular Magnetic Resonance 2012 14(Suppl 1):O40.

Submit your next manuscript to BioMed Central and take full advantage of:

- Convenient online submission

- Thorough peer review

- No space constraints or color figure charges

- Immediate publication on acceptance

- Inclusion in PubMed, CAS, Scopus and Google Scholar

- Research which is freely available for redistribution

Submit your manuscript at www.biomedcentral.com/submit
C Biomed Central 